\title{
Territorialité politique, nationalisme et traversées constitutionnelles en Écosse
}

Territoriality, Nationalism and Constitutional Crossings

\section{Arnaud Fiasson}

\section{(2) OpenEdition}

12 Journals

Édition électronique

URL : http://journals.openedition.org/etudesecossaises/1069

DOI : 10.4000/etudesecossaises.1069

ISSN : 1969-6337

Éditeur

UGA Éditions/Université Grenoble Alpes

\section{Édition imprimée}

Date de publication : 25 avril 2016

Pagination : $51-67$

ISBN : 978-2-84310-324-7

ISSN : $1240-1439$

Référence électronique

Arnaud Fiasson, «Territorialité politique, nationalisme et traversées constitutionnelles en Écosse », Études écossaises [En ligne], 18 | 2016, mis en ligne le 01 janvier 2017, consulté le 15 mars 2021. URL: http://journals.openedition.org/etudesecossaises/1069; DOI : https://doi.org/10.4000/ etudesecossaises.1069 


\section{Territorialité politique, nationalisme et traversées constitutionnelles en Écosse}

À l'occasion de la cérémonie qui a officiellement marqué, le $1^{\text {er }}$ juillet 1999, l'ouverture du parlement écossais, Donal Dewar déclare que la mise en place de la dévolution législative a donné un nouvel élan à la nation écossaise :

This is a moment anchored in our history. Today, we reach back through the long haul to win this Parliament, through the struggles of those who brought democracy to Scotland, to that other Parliament dissolved in controversy nearly three centuries ago. [...] But today there is a new voice in the land, the voice of a democratic Parliament. A voice to shape Scotland, a voice for the future. ("A Moment Anchored in History», $B B C$ UK Nerws, 1999)

La mise en place des institutions autonomes, telles que le parlement et le gouvernement écossais, marque la création d'une arène politique écossaise dans laquelle évoluent des acteurs qui lui sont propres et qui légifèrent, dans la limite des pouvoirs dévolus, sur les affaires écossaises (Keating, 2001, p. 205). La dévolution législative, dont l'objet reste le renforcement de l'union politique et économique qui cimente le RoyaumeUni, a donc permis, dans le cas de l'Écosse, de politiser un territoire dont les frontières avaient été entretenues de manière implicite par la société civile écossaise qui conservait ses institutions ecclésiastiques, judiciaires et scolaires depuis l'Union de 1707 (Civardi, 1998, p. 62-63). Fort d'une majorité absolue au sein d'une institution parlementaire qui a pourtant fait de la notion du partage du pouvoir l'un de ses principes fondateurs, le Scottish National Party (SNP) occupe une position politique privilégiée qui lui permet d'avancer son agenda indépendantiste.

Cet article, dont les fondements théoriques s'appuient sur le modèle de territorialité développé par Jan Penrose (2002), étudie la place qu'occupe le territoire politique écossais dans la construction de l'idéologie nationaliste promue par le SNP. Le regard que nous portons sur la relation centre-périphérie au sein du système politique britannique ambitionne de démontrer que le SNP exploite l'espace politique écossais délimité par 
le Scotland Act 1998 de manière à construire un argumentaire nationaliste qui minimise l'affirmation de la dimension culturelle de l'identité écossaise au profit de la revendication de l'ensemble des leviers politiques et économiques nécessaires au gouvernement d'une Écosse indépendante.

\section{Pratiques du modèle de territorialité}

Robert Sack conçoit la territorialité ainsi : «the attempt by an individual or group to affect, influence or control people, phenomena and relationships by delimiting and asserting control over a geographic area [...] called a territory» (1986, p. 19). La géographe Jan Penrose s'appuie sur cette définition et propose le modèle d'une stratégie géographique qui connecte la société à l'espace, faisant alors de la territorialité l'expression géographique de la notion de pouvoir. Elle établit le postulat selon lequel la territorialité correspond au processus d'agencement que l'humain fait de l'espace en le transformant en territoire. Les territoires, quant à eux, possèdent une caractéristique fondamentale : ils sont délimités par des frontières. Ces frontières, qui sont de natures diverses (historique, naturelle, culturelle, politique, économique), restent alors libres d'être exploitées de manières différentes dans la construction de la territorialité (Paasi, 1995, p. 42). Plus particulièrement, Penrose indique :

$[W]$ hen people create territories, they create boundaries that both unite and divide space along with everything that it contains. By combining some people and certain resources and separating them from other people and other resources, the creation of territories gives physical substance and symbolic meaning to notions of "us" and "them" and "ours" and "theirs". (Penrose, 2002, p. 180)

La géographe prolonge son modèle de territorialité par un procédé de construction identitaire qui repose sur deux pouvoirs qu'elle caractérise de latents : un pouvoir matériel, qui accorde la possibilité d'exploiter des ressources existantes et qui permet à l'humain de survivre, et un pouvoir émotionnel qui permet aux frontières du territoire de subsister, notamment en raison de l'attachement des individus à des lieux spécifiques, mais également par références à l'histoire, à la mémoire et à des mythes communs. Ce procédé de construction identitaire s’inscrit donc dans la veine de la «communauté politique imaginée» théorisée par Benedict Anderson et évoque le «nationalisme banal» repéré par Michael Billig (Anderson, 1990, p. 15-16; Billig, 1995, p. 6-9).

Penrose présente deux formes de territorialité. La première, selon laquelle l'identité est culturellement définie, a pour frontière la distribution culturelle de l'espace et privilégie l'attachement émotionnel d'un groupe 
à son territoire. Les ressources matérielles ont une place importante mais secondaire : elles renforcent le pouvoir émotionnel. À l'inverse, lorsque l'identité est territorialement définie, les frontières sont formées par la distribution géographique d'une unité politique. Cette forme de territorialité privilégie le contrôle des ressources naturelles en vue de constituer le territoire et les stratégies pour le maintenir. L'émotionnel a une place secondaire dans laquelle le pouvoir matériel puise pour se renforcer.

$\mathrm{Au}$ sein des études écossaises, le rôle joué par la confluence du pouvoir émotionnel et du pouvoir matériel dans les processus de construction identitaire et territoriale de l'Écosse est présent dans plusieurs travaux universitaires. Selon Cairns Craig, l'imaginaire national est un espace dans lequel a cours un dialogue qui définit les particularités de l'expérience humaine au sein d'un territoire dont les frontières définissent les limites selon lesquelles certaines voix, passées et présentes, sont entendues mais auxquelles on résiste (1999, p. 31). Dans une veine similaire, Richard Finlay rappelle que nombre de mythes écossais ont joué un rôle conséquent dans le développement politique, social et culturel de la nation, soit en tant qu'opérateur de changement, soit en vue d'exprimer une idéologie politique dominante (1997, p. 123). Ainsi, les symboles de l'identité écossaise, qui ne se limitent pas à Bruce, Bannockburn et Burns, sont utilisés et réinterprétés afin de servir l'élaboration de valeurs spécifiques à la nation écossaise (Reicher et coll., 2009, p. 38). Robert Crawford reconnaît également que la mythologie, l'imaginaire et la littérature font partie intégrante d'un processus historique, mais il ajoute que l'imagination d'une Écosse indépendante par le lecteur garantit à ce dernier une «soupape de sécurité» («safety valve») qui permet d'échapper aux réalités du présent immédiat (2014, p. 1-2, p. 8). Alan Riach semble renforcer cette position lorsqu'il soutient :

[The cultural argument] was there long before North Sea oil was discovered, and it will be here long after the oil has run out.

It is the only distinction that matters. No-one denies the importance of economics - putting bread on the table, jobs and health — but they are all matters of material fact unless occupied and enlivened by imagination. The arts - music, painting, architecture and, preeminently, literature - are the fuel and fire that makes imagination possible. Neglect them at your peril. (Moffat \& Riach, 2014, p. 25)

Par ailleurs, Riach affirme que, sans les œuvres de William Dunbar, Robert Burns, Hugh MacDiarmid ou encore Liz Lochhead (pour ne citer que quelques auteurs), l'Écosse ne serait rien de plus qu'une entité géographique qui resterait alors libre d'être exploitée (Moffat \& Riach, 2014, p. 212). Par conséquent, les universitaires précédemment cités rejoignent la première forme de territorialité établie par Penrose : le pouvoir matériel 
cède le pas au pouvoir émotionnel au sein d'un processus de construction du territoire écossais qui repose sur une identité nationale définie culturellement.

À rebours de cette pratique de la territorialité, le SNP affirme que l'autodétermination ne relève pas d'un attachement émotionnel au territoire écossais : il s'agit de contrôler l'ensemble des leviers politiques qui régissent la totalité des ressources présentes en Écosse. À titre d'exemple, nous retenons les propos de Nicola Sturgeon, alors numéro deux du gouvernement SNP, prononcés lors d'un discours qu'elle présente le 25 janvier 2013 devant la Chambre de commerce britanno-irlandaise.

Contrary to what many outside observers might imagine, the debate we are having is not about national identity. [...] My argument is that with the full range of economic and social powers in the hands of the Scottish Parliament, we will be better able to achieve the fairer and more prosperous society that we aspire to. As an independent country, the Scottish Government will have the range of tools that will better equip us to build on our economic strategy and produce even better results. (Sturgeon, 2013)

Selon cette vision, réitérée en particulier lors de la campagne référendaire de 2014, l'argumentaire nationaliste s’inscrit dans la lignée de la seconde pratique de la territorialité établie par Penrose. Le pouvoir matériel préside au pouvoir émotionnel au sein d'un processus de construction du territoire écossais qui allie l'identité politique de l'Écosse à la légitimation d'une institution nationale (le Parlement écossais) et qui met potentiellement en jeu la stabilité de l'État britannique.

Avant de procéder à la validation du caractère opérationnel du modèle théorique de territorialité de Penrose appliqué au cas écossais, il importe de pondérer la dichotomie opérée, dans une démarche analytique, entre le pouvoir émotionnel et le pouvoir matériel. À cet effet, le tableau nuancé que présente Robert Thomsen fait apparaître un nationalisme polymorphe qui ne se restreint pas à la distinction artificielle entre les deux pratiques de la territorialité énoncées précédemment.

Cultural and political nationalism should not be seen as irreconcilable oppositions but as 'ideal' positions at either end of the same continuum. These ideal positions rarely exist in the real world. Rather, cultural nationalism often develops into a nationalism of a more political kind. Similarly, political nationalism may recede into a more cultural, apolitical form. If we think of the two types of nationalism not as separate entities but as continuations of each other, the distinction is useful in the analysis of the development of autonomism. (Thomsen, 2013, p. 6)

La longueur du présent article ne permettant pas d'analyser en profondeur la convergence des deux formes de territorialité dans le processus de territorialisation de l'espace politique écossais, nous focalisons 
notre étude sur la place qu'occupe le territoire politique écossais dans la construction de l’idéologie nationaliste promue par le SNP.

\section{L'institution parlementaire écossaise : spécificités territoriales d'une traversée constitutionnelle}

Le Scotland Act $1998^{1}$ définit les frontières du territoire politique écossais et rend compte de l'étendue de la traversée constitutionnelle de la frontière anglo-écossaise qui s'opère dès l'ouverture de l'institution parlementaire écossaise. Cette traversée constitutionnelle s'accompagne donc d'une traversée géographique puisqu'elle place Édimbourg, et non Londres, en tant que capitale de l'Ecosse où résident les pouvoirs législatifs et exécutifs qui régissent le gouvernement du territoire écossais. Il est alors possible de considérer le Scotland Act 1998 comme l'opérateur de la territorialité puisqu'il permet la redéfinition de la notion de pouvoir au Royaume-Uni. Dans le cas de l'Écosse, les deux niveaux de pouvoir recouvrent d'une part les sujets réservés à Westminster et d'autre part les sujets dévolus au parlement écossais. Afin de procéder à l'identification des pouvoirs concédés au parlement écossais par Westminster, il semble préférable de réfléchir en termes de «compétence législative», comme en témoigne cet extrait de l'alinéa 29 :

(1) An Act of the Scottish Parliament is not law so far as any provision of the Act is outside the legislative competence of the Parliament.

(2) A provision is outside that competence so far as any of the following paragraphs apply-

(a) it would form part of the law of a country or territory other than Scotland, or confer or remove functions exercisable otherwise than in or as regards Scotland,

(b) it relates to reserved matters.

Cet alinéa, qui réfêre à l'impossibilité inhérente au parlement écossais de légiférer sur des lois qui ne touchent pas l'Écosse, met en exergue une différence fondamentale entre les deux institutions parlementaires : le principe de souveraineté parlementaire retenu par Westminster. Alors que Westminster dispose du droit de voter des lois applicables au RoyaumeUni (et donc à l'Écosse), le parlement écossais ne peut légiférer sur des aspects non écossais ${ }^{2}$, c'est-à-dire anglais, gallois ou nord-irlandais. Cette

1. L'intégralité du texte législatif est disponible sur <http://www.legislation.gov.uk/ukpga/1998/ 46/pdfs/ukpga_19980046_en.pdf>.

2. On garde à l'esprit la portée de la territorialisation soulevée par la controversée West Lothian Question, puisque les députés au parlement britannique élus dans des circonscriptions écossaises 
incapacité législative s'étend également aux sujets réservés à Westminster. Sous l'appellation «reserved matters», l'annexe 5 du Scotland Act 1998 définit en intégralité les sujets réservés que l'on peut regrouper selon les catégories suivantes : la Constitution, la défense nationale, les affaires étrangères, la sécurité sociale, la gestion de la macro-économie et l'immigration. À l'inverse, les sujets dévolus correspondent à tout sujet qui n'est pas circonscrit par le Scotland Act 1998. On y trouve par exemple l'agriculture, la santé, l'environnement, les services d'ordre, et le transport. Les sujets réservés à Westminster et les sujets dévolus au parlement écossais constituent donc autant de points de repères qui balisent la notion de traversée constitutionnelle instaurée par le texte fondateur du parlement écossais et remaniée au rythme des amendements portés sous la forme du Scotland Act 2012 et du Scotland Bill 2015-20163.

La territorialisation de l'espace politique écossais s'effectue dans la continuité des traditions parlementaires britanniques et alimente l'argument d'une Écosse perçue comme une nation «sans État» (Brown et coll., 1998, p. 27). L'alinéa 28 indique que les lois suivent le même parcours général : le projet de loi (Bill) doit d'abord être présenté devant les députés du parlement écossais (Members of the Scottish Parliament ou MSPs) pour être débattu et approuvé par un vote. Ce projet ne peut devenir loi (Act of the Scottish Parliament ou ASP) qu'après validation par le monarque sous la forme de la sanction royale (Royal Assent). On remarque également que l'alinéa 6 témoigne du caractère juridique que prend la mise en application d'une loi. De plus, l'alinéa 7 stipule que le parlement britannique reste souverain, c'est-à-dire dire qu'il dispose du droit d'abroger toute loi du parlement écossais et de l'obliger à entériner les siennes. Sous l'autorité de l'alinéa 84, les candidats élus au parlement écossais s'engagent à prêter serment d'allégeance, faute de quoi ils commettraient une infraction et ne pourraient être autorisés à exercer leur fonction. Bien que ce serment puisse être prêté dans une langue autre que l'anglais, le fait qu'il constitue une nécessité s'inscrit dans la continuité des traditions parlementaires et monarchiques britanniques, si ce n'est des traditions anglaises. Par ailleurs, le rôle joué par le président de séance au parlement écossais (Presiding Officer) ressemble fortement à celui du Speaker dans la Chambre des communes ainsi qu’à celui du président

peuvent exercer leur droit de vote sur des lois applicables uniquement en Angleterre, au pays de Galles, ou en Irlande du Nord. À l'inverse, les députés au parlement britannique ne peuvent pas voter des lois relevant de la compétence législative des institutions dévolues, à moins d'y être également élus.

3. Au stade de l'écriture du présent article, le Scotland Bill 2015-2016 fait l'objet d'un examen auprès de la commission parlementaire britannique. Aucune date n'est encore prévue pour le passage du projet de loi en troisième lecture. 
du parlement européen. Le président de séance est également en charge de contrôler le bien fondé du texte de loi et il doit s'assurer que le projet législatif relève des compétences transférées au parlement écossais, avant de soumettre ensuite le projet pour obtention de la sanction royale. Par ailleurs, le rôle joué par l'exécutif écossais (devenu Gouvernement écossais en 2007) se rapproche de celui joué par le Cabinet britannique ${ }^{4}$. L'utilisation de la formule de Barnett, mise en place en 1979, perdure aujourd'hui encore, même si la dotation forfaitaire que verse la trésorerie britannique aux composantes du Royaume-Uni fait toujours l'objet d'un débat (Barnes, 2013; Maddox, 2013).

L'affirmation des traits spécifiques à l'institution parlementaire nationale écossaise renforce le caractère distinctif du territoire politique écossais qui s'érige au sein du Royaume-Uni. Les analogies entre les parlements témoignent de la nature «hybride» de l'institution écossaise. En effet, ces similitudes, dans le sens de ressemblances partielles et non totales, ne participent pas à la constitution de deux institutions identiques. Le parlement dispose d'un système électoral qui lui est propre : tandis que l'élection des membres dans les circonscriptions écossaises s'effectue selon le scrutin majoritaire à un tour traditionnel (first-past-the-post), l'apparition d'une forme de proportionnalité dans le vote régional (additional member system) constitue une spécificité bien particulière aux élections parlementaires écossaises (Scotland Act 1998, art. 1, art. 7). Chaque système de vote octroie respectivement 73 et 56 sièges, soit un total de 129 députés. Cela a permis, par ailleurs, l'émergence de petits partis qui ne trouvaient pas leur place à Westminster ${ }^{5}$. C'est d'ailleurs cette diversité politique présente en 2003 qui valut au parlement écossais l'appellation rainbow parliament, chaque parti étant représenté par une couleur. Exception faite de l'élection législative du 5 mai 2011, il est important de noter que cette forme de proportionnalité fut conçue afin qu'aucun parti (en particulier le SNP) ne dispose d'une majorité parlementaire dans l'institution écossaise, par opposition aux majorités fréquentes à Westminster (Bradbury, 2006, p. 577). Par ailleurs, le nombre de femmes élues au parlement écossais a constamment été supérieur à celui de Westminster, même si des efforts restent à entreprendre en vue d'une parité absolue. La dynamique plus consensuelle de la politique écossaise se retrouve élégamment dans la disposition de la Chambre des débats. À la différence du parlement

4. Contrairement au cadre britannique, le Presiding Officer et le First Minister sont élus par le parlement écossais.

5. On compte, parmi les forces politiques représentées dès l'élection de 1999, le Parti travailliste, le SNP, le Parti libéral-démocrate, le Parti conservateur, les Verts, le Scottish Socialist Party et un député indépendant. 
britannique, le parlement écossais est monocaméral et il est disposé en hémicycle, à la manière des parlements français et européen, ce qui contraste fortement avec le climat d'opposition qui se reflète dans la disposition de la Chambre des communes. De plus, tandis que le parlement écossais s'inscrit dans la tradition de la démocratie représentative, l'institution présente toutefois certaines innovations qui relèvent d'un modèle participatif spécifique à l'Écosse. Le parlement écossais se doit de donner suite à toute pétition jugée recevable et en toute transparence, à l'inverse de Westminster (Camp-Pietrain, 2008, p. 3). Par ailleurs, et au-delà de la forme de proportionnalité amenée par le scrutin mixte lors des élections écossaises, les organisations de la société civile sont plus aisément intégrées aux affaires parlementaires et l'institution écossaise fonctionne à des horaires qui se veulent compatible avec la vie de famille. À la manière dont les spécificités du parlement écossais renforcent la notion de territorialité institutionnelle, le cadre juridique dessiné par le Scotland Act 1998 fait apparaître un modèle parlementaire "'étranger' et [qui] représente de ce fait une menace pour les tenants de la tradition parlementaire britannique» (Duclos, 2009, p. 5, 13).

À la lumière de l'étude du cadre institutionnel écossais mis en place dans l'objectif de renforcer l'union politique qui cimente les parties constituantes du Royaume-Uni, nous arguons que le territoire politique écossais sert de plateforme à l'agenda indépendantiste et socio-démocratique du SNP qui fait de la territorialité le fondement de son argumentaire nationaliste.

\section{La territorialité en pratique : le SNP à l'exercice du pouvoir politique}

Les aires géographiques que constituent les circonscriptions et les régions d'Écosse constituent également un vecteur de la territorialisation de l'espace politique. En effet, le territoire politique écossais, composé de l'ensemble de ces aires géographiques, est régi par le parlement et le gouvernement écossais qui contrôlent, dans la limite des compétences législatives qui leur sont dévolues, l'exploitation des ressources humaines et matérielles en Écosse. Si les suffrages exprimés lors des élections législatives écossaises représentent les préférences des Écossais en matière de gouvernement, ils témoignent également d'une affirmation identitaire de l'électorat en termes politiques :

When asked in devolved elections to consider who and what they think would be best for Scotland, some people persistently give a different answer than when they are asked, as in UK general elections, who can best govern the UK as a whole. In particular, the answer 
they give to the question posed by devolved elections has persistently been more likely to be 'the SNP', both as a way of expressing their distinctive Scottish identity and of ensuring that they have a devolved government willing to stand up for Scotland's particular interests. (Curtis et coll., 2009, p. 184)

Comme l’indique la représentation cartographique des résultats aux élections législatives écossaises de 1999 et 2011 (fig. 1; fig. 2; Ordnance Survey, 2007, p. 1; SPICe, 2011, p. 33), les suffrages exprimés en faveur du SNP renforce la dimension territoriale l'espace politique écossais.

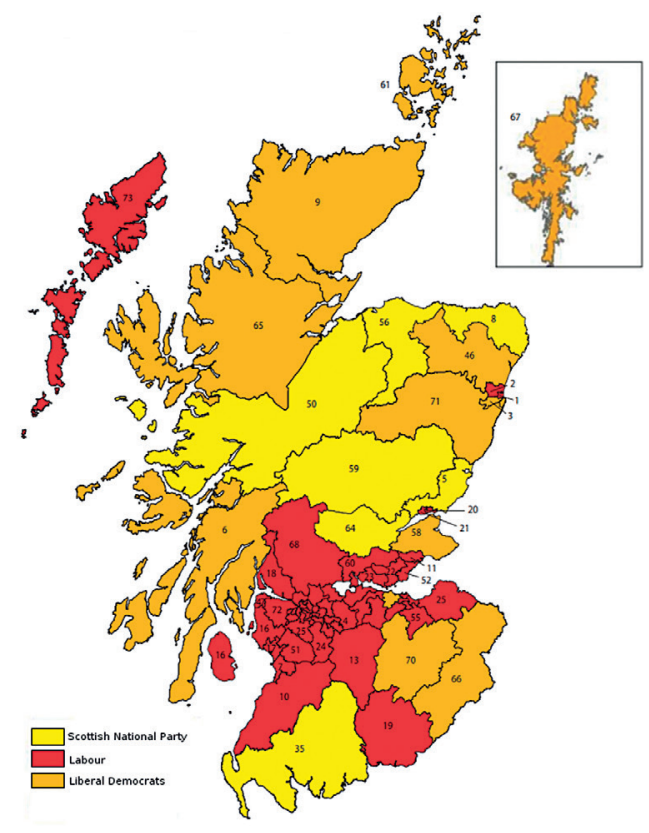

Figure 1. - Constituency seats by Party 1999.

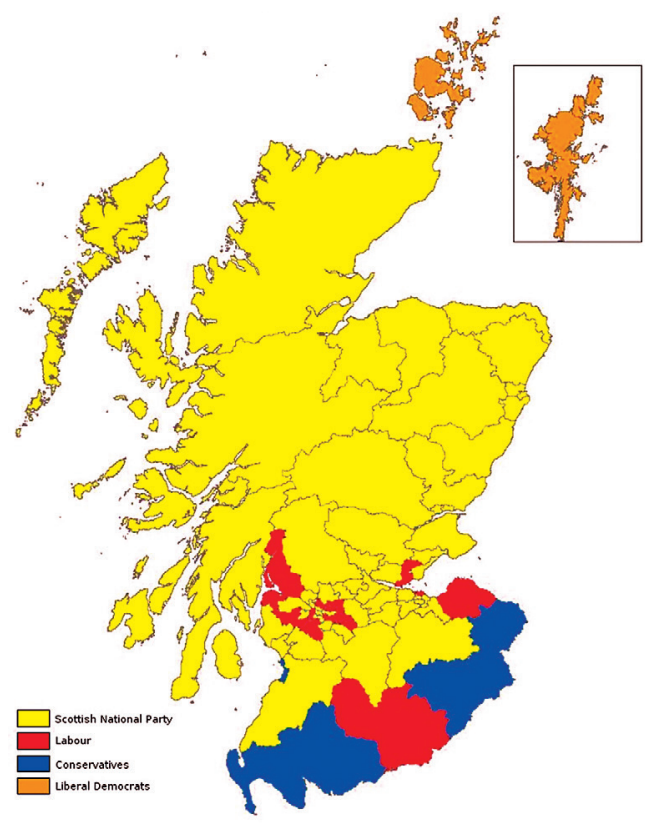

Figure 2. - Constituency seats by Party 2011.

L’arène politique spécifiquement écossaise que constitue Holyrood, par opposition à la dimension britannique inhérente à Westminster, a permis l'émergence du SNP au rang de force politique significative. En effet, alors que le SNP ne remporte pas plus de six sièges à Westminster depuis 1979 (exception faite de l'élection législative de 2015), il constitue le premier parti d'opposition lors des deux premières législatures écossaises. Il obtient ensuite une majorité relative au sein du parlement écossais en 2007, et une majorité absolue en 2011. Un bref regard sur les deux administrations SNP montre toutefois que la vive progression du parti nationaliste sur le territoire électoral national écossais en l'espace d'une dizaine d'années émane du pragmatisme dont fait preuve le SNP à l'égard de l'espace politique créé par le Scotland Act 1998. 
D'une part, la victoire du SNP en 2007 provient en partie du vote protestataire à l'encontre des politiques du Parti travailliste, tant au niveau britannique qu'écossais, et ne traduit pas une résurgence des velléités indépendantistes de l'électorat (Curtis et coll., 2009). Par ailleurs, si l'administration SNP est en mesure de défendre les intérêts écossais par le vote du Abolition of Bridge Tolls (Scotland) Act 2008 et du Graduate Endowment Abolition (Scotland) Act 2008, elle ne parvient pas, en raison de sa nature minoritaire, à matérialiser certaines des promesses phares de son programme électoral (SNP, 2007), telles que la loi sur le prix minimal par unité d'alcool (minimum pricing on alcohol) ${ }^{6}$. L'administration SNP reste donc tributaire d'une quête de consensus au sein de l'institution parlementaire.

D'autre part, il convient de nuancer l'étendue territoriale de la victoire électorale remportée par le SNP en 2011. La majorité des votes par circonscription et par région que le SNP remporte ne représente que $44 \%$ des suffrages exprimés, soit, au pro rata d'un taux de participation de 50,4\%, moins d'un quart de l'électorat inscrit. Par ailleurs, et contrairement à l'élection de 2007, la forme de proportionnalité amenée par le scrutin de liste au niveau régional n'est pas l'unique responsable de l'accession du parti au pouvoir :

Evidently the First Past the Post element of the electoral system exhibited a bias against the SNP that was not in evidence in 2007. The party has frequently found it more diffcult than Labour to win seats under First Past the Post because its vote is geographically more evenly distributed, with the result that the party tends to collect many second places and relatively ferw firsts. (Curtice \& Steven, 2011, p. 16)

Parmi les circonstances favorables aux gains électoraux du SNP, on compte, entre autres, la crédibilité acquise par l'administration SNP au cours des quatre années précédentes, le charisme d'Alex Salmond et la stratégie positive du SNP qui l'emporte face à la négativité du travailliste Iain Gray lors de la campagne électorale, l'exploitation des sites de réseaux sociaux par le parti indépendantiste et la transformation de ce dernier en machine de campagne électorale (Duclos, 2013; Leydier, 2013).

Plutôt que fonder son idéologie nationaliste sur une définition ethnique de l'identité écossaise, c'est-à-dire sur des caractéristiques partagées et déterminées par la langue (la défense du gaélique et de l'écossais n'a jamais constitué une priorité), la religion ou l'ethnicité, le SNP préfère

6. Le projet de loi présenté par la secrétaire à la Santé Nicola Sturgeon, introduisant un prix minimum de 45 pence par unité d'alcool, est rejeté dès le premier stage par la commission parlementaire qui préfère laisser la législation aux mains de Westminster. L'opposition est constituée des travaillistes, des conservateurs et des libéraux-démocrates (Currie, 2010, p. 6). 
une conception civique et volontariste de la nationalité qui repose sur des valeurs et des institutions communes. À Nicola Sturgeon de déclarer :

[T] he fact of nationhood or Scottish identity is not the motive force for independence. Nor do I believe that independence, however desirable, is essential for the preservation of our distinctive Scottish identity. And I don't agree at all that feeling British - with all of the shared social, family and cultural heritage that makes up such an identity-is in any way inconsistent with a pragmatic, utilitarian support for political independence.

(Sturgeon, 2012)

À partir des années 1990, et plus particulièrement suite aux élections législatives européennes de 2014 qui se sont traduites par une percée de l'extrême-droite, le SNP a été soucieux de projeter une image inclusive du nationalisme écossais (Lynch, 2002, p. 211-214). Ainsi, tandis que le SNP privilégie la formulation «the people who live and work in Scotland» à celle de «Scottish people» (Duclos, 2014, p. 139-152), le livre blanc Scotland's Future: Your Guide to an Independent Scotland publié en amont du référendum de 2014 exemplifie la volonté du SNP d'établir son argumentaire sur la base de faits rationnels plutôt qu'émotionnels. Il s'agit de s'écarter d'une identité nationale réduite à ses caractéristiques culturelles afin de recentrer le débat concernant la question nationale sur le statut politique de l'Écosse et sur les pouvoirs octroyés à la structure parlementaire nationale placée en charge des décisions politiques et économiques régissant le fonctionnement du territoire écossais. Par ailleurs, l'intransigeance du SNP à l'égard de la constitution du corps électoral participant à la consultation référendaire de 2014 témoigne d'une pratique du principe de territorialité qui fait du critère de résidence sur le territoire écossais le point d'ancrage de l'identité nationale civique que le parti politique promeut.

L'identité écossaise proposée par le SNP ne repose donc pas sur une définition exclusivement culturelle : elle relève de l'exploitation des ressources humaines et matérielles au sein de l'entité géopolitique que représente l'Écosse et est animée par le panachage d'un idéal sociodémocratique et d'une volonté de vivre ensemble au sein du territoire écossais qui se démarque des autres parties constituantes du RoyaumeUni (MacAskill, 2004, p. 41-42). Lors de la campagne référendaire, les nationalistes présentent leur argumentaire aussi bien comme une réponse à une demande sociale que comme une critique du pouvoir politique au Royaume-Uni. Tandis que le SNP met l'accent sur la crédibilité qu'il a acquise en matière de parti au gouvernement, il se targue de représenter le parti le plus à même de défendre les intérêts de l'Écosse, par opposition au gouvernement de coalition mis en place en 2010 entre les partis conservateur et libéral-démocrate. Le SNP invoque ainsi l'emprise du déficit démocratique dont l'Écosse a particulièrement souffert lors des 
législatures Thatcher et Major de 1979 à $1997^{7}$. Plus particulièrement, il estime que l'indépendance n'ouvrirait pas seulement la voie à une gestion plus adaptée aux besoins de l'Écosse, mais offrirait l'occasion de se démarquer de la politique d'austérité et des coupes budgétaires promues par le gouvernement britannique. Le SNP ne manque alors pas de rappeler qu'à la différence de la Poll Tax votée par le parlement britannique et imposée à l'Écosse en 1989, la Bedroom Tax a été contrecarrée par le parlement écossais qui fut en mesure de limiter les effets de cet impôt. Les indépendantistes disposent également d'un argument de taille en regard de la présence d'armes nucléaires sur le sol écossais. Le gouvernement britannique, qui entend renouveler son arsenal nucléaire, a estimé que le coût d'une telle politique pourrait s'élever à 31 milliards de livres sterling. Lors d’un débat télévisé qui se déroule dans le cadre la campagne référendaire, Alex Salmond affirme qu'une Écosse indépendante refuserait la présence d'armes de destruction massive sur son territoire et qu'elle privilégierait la réduction des inégalités sociales et le maintien de l'hôpital public — actuellement menacé par d'importantes coupes budgétaires et par la pression qu'exerce la privatisation des services publics en Angleterre. Le SNP entend donc convaincre l'électorat que l'indépendance permettrait de mettre un terme à la division Nord-Sud, qui correspond à la représentation géographique des divergences politiques, économiques et sociales qui séparent le Sud-Est de l'Angleterre (dont Londres) du reste du Royaume-Uni. Parallèlement, le SNP soutient que l'Écosse deviendrait le premier producteur pétrolier de l'Union européenne - elle disposerait de $60 \%$ du total des ressources pétrolières européennes - et qu'elle obtiendrait la deuxième place en termes de réserves gazières, après les Pays-Bas (Scottish Government, 2013a, p. 301). Ainsi, une territorialité d'ordre économique s'ajoute à la territorialité géopolitique pratiquée par le SNP.

Le nationalisme écossais occupe une place singulière sur la scène politique européenne et internationale. En effet, contrairement aux référendums concernant l'émancipation de la Crimée et l'avenir constitutionnel de la Catalogne qui se sont tenus respectivement le 16 mars et le 9 novembre 2014, le référendum écossais a été salué pour son caractère procédurier et démocratique. Loin de la violence des affrontements et des modalités contestables du scrutin en Crimée, et à la différence du gouvernement autonome catalan qui n'envisageait pas d'obtenir l'approbation du gouvernement central espagnol, le SNP choisit de se plier au

7. Cette stratégie se veut renforcer la spécificité du territoire politique écossais puisque, au Xxi ${ }^{e}$ siècle encore, les dix-huit années de règne du Parti conservateur sont perçues comme un traumatisme qui a laissé une marque sur l'identité écossaise (Maddox, 2010, p. 1). 
cadre législatif et ne considère pas le mandat électoral qu'il obtient à l'issue des élections législatives écossaises de 2011 comme suffisant à déclarer l'indépendance de l'Écosse. Dans la mesure où la Constitution britannique fait partie des compétences réservées à Westminster, le SNP applique la stratégie référendaire qu'il avait fermement défendue dès le lancement d'une Conversation nationale (National Conversation) en 2007 et lors de la campagne électorale de 2011 (Scottish Executive, 2007, p. 38; SNP, 2011, p. 28). À l'issue de négociations avec le gouvernement britannique, l'accord d'Édimbourg (Edinburgh Agreement) du 15 octobre 2012 confère au parlement écossais le pouvoir d'organiser le référendum, entérine la légalité de celui-ci, et engage les gouvernements écossais et britannique à se plier à son résultat. Dès lors, l'inscription d'une Écosse indépendante au sein du concert des États-nations européens et internationaux que le SNP propose ne déroge pas à l'importance que retient la notion de territoire dans la construction de son argumentaire nationaliste. En effet, et puisqu'il n'existe pas de précédent comparable à la situation écossaise dans les traités européens, le SNP profite de ce vide juridique et invoque l'article 48 du Traité sur l'Union européenne (Scottish Government, 2013a, p. 221). Le gouvernement écossais affirme alors qu'une réponse favorable au référendum se traduirait par la création de deux États successeurs, l'Écosse et le reste du Royaume-Uni, dont l'adhésion à l'Union européenne serait automatique ${ }^{8}$ bien que sujette à des renégociations tenant compte des réagencements territoriaux. Par ailleurs, si le SNP propose une transition vers l'indépendance qui maintiendrait la libre circulation au sein des îles Britanniques, il n'envisage pas l'entrée de l'Écosse dans l'espace Shengen qui se traduirait par l'abattement des frontières du territoire (Scottish Government, 2013a, p. 223). Au plan international, le rejet de la présence d'armes nucléaires sur le territoire écossais, que le SNP promet d'inscrire dans la Constitution d'une Écosse indépendante (Scottish Government, 2013b, p. 9), accentue la territorialisation d'un espace politique écossais qui laisse toutefois planer un doute quant à l'adhésion éventuelle d'une Écosse indépendante à l'Organisation du traité de l'Atlantique nord (Duclos, 2014, p. 250-252).

Tandis que le parlement d'Holyrood s'impose comme l'institution clé via laquelle s'érige aujourd'hui encore la territorialisation de l'espace politique écossais, ses spécificités institutionnelles ont permis l'accession

8. Sous la pression de l'opposition parlementaire écossaise qui affirme que le reste du RoyaumeUni deviendrait le seul État successeur en vertu de l'article 49, le SNP revient sur sa déclaration en octobre 2012 lorsqu'il admet ne pas disposer de confirmation juridique au sujet d'une adhésion automatique de l'Écosse à l'Union européenne (Duclos, 2014, p. 241). 
du SNP au rang de parti majoritaire. Le nationalisme civique que prône le SNP participe à la définition d'une identité politique qui privilégie le contrôle des leviers économiques et politiques nécessaires à la gestion d'une Écosse indépendante. Au-delà d'une pratique de la territorialité alignée selon un agenda socio-démocratique qui se distingue des politiques en vigueur dans le reste du Royaume-Uni, le SNP a porté sur le devant de la scène internationale ses revendications indépendantistes proeuropéennes qui restent de mise à l'approche du référendum prévu par le gouvernement conservateur en ce qui concerne la sortie du RoyaumeUni du giron européen.

Paradoxalement, si le référendum de 2014 s'est traduit par l'échec des nationalistes, il marque également le point de départ de traversées constitutionnelles supplémentaires et favorise la territorialisation des différents espaces politiques au sein du Royaume-Uni. Lorsqu'il porte les revendications autonomistes anglaises au cri de «English Vote for English Laws» au lendemain du référendum, David Cameron reconnaît que le Royaume-Uni traverse une crise politique et propose de la résoudre par l'intermédiaire de la commission Kelvin placée en charge d'examiner les modalités d'un accroissement des compétences législatives au sein de chaque partie constituante du Royaume-Uni. Cinq mois après la publication du rapport de la commission, le SNP remporte 56 des 59 sièges alloués à l'Écosse lors de l'élection législative britannique. Au-delà du caractère exceptionnel de ce résultat, la victoire du SNP semble indiquer que le phénomène de territorialisation ne se limite pas à l'espace politique créé par le Scotland Act 1998. Il s'étend à l'espace politique britannique instauré par l'Acte d'Union de 1707 qui octroie à l'Écosse une représentation parlementaire au sein du parlement britannique.

\section{Bibliographie}

\section{Articles de presse}

«A Moment Anchored in History», BBC UK News, 1999 ( $1^{\text {er }}$ juillet). Disponible sur <http://news.bbc.co.uk/2/hi/special_report/1999/ 06/99/scottish_parliament_opening/383029.stm>.

BARnEs Eddy, 2013 (3 novembre), «Welsh First Minister Urges Barnett Formula Rethink», The Scotsman. Disponible sur <www.scotsman. $\mathrm{com} / \mathrm{news} / \mathrm{uk} /$ welsh-first-minister-urges-barnett-formula-rethink$1-3170833>$.

Currie Brian, 2010 ( $1^{\mathrm{er}}$ septembre), «Calls for Standard Price Limit on Alcohol across UK», The Glasgow Herald, p. 6. 
Maddox David, 2010 (15 mai), «Back to the '80s», The Scotsman, p. 1.

_ 2013 (18 décembre), «Barnett Formula Would Remain after No

Vote», The Scotsman. Disponible sur <http://www.scotsman.com/ news/politics/top-stories/barnett-formula-would-remain-after-novote-1-3238320>.

\section{Sources primaires}

Consultative Steering Group, 1999, Shaping Scotland's Parliament: Report of the Consultative Steering Group on the Scottish Parliament, Édimbourg, Stationery Office.

Ordnance Survey, 2007, The Scottish Parliament 1999 Election Results, Édimbourg, Scottish Parliament Corporate Body. Disponible sur $<$ www.scottish.parliament.uk/EducationandCommunityPartner shipsresources/1999_election_results.pdf>.

Scotland Act 1998, Chapter 46, Londres, The Stationery Office. Disponible sur <www.legislation.gov.uk/ukpga/1998/46/pdfs/ukpga_1998 0046_en.pdf $>$.

Scotland Act 2012, Chapter 11, Londres, The Stationery Office. Disponible sur <www.legislation.gov.uk/ukpga/2012/11/pdfs/ukpga_2012 $0011 \_$en.pdf $>$.

Scottish Constitutional Gonvention, 1995, Scotland's Parliament, Scotland's Right, Édimbourg, Scottish National Convention.

Scottish Executive, 2007, Choosing Scotland's Future: A National Conversation, Édimbourg, Scottish Executive.

Scottish Government, 2013a, Scotland's Future: Your Guide to an Independent Scotland, Édimbourg, Scottish Government.

_ , 2013b, Scotland's Future: From the Referendum to Independence and a Written Constitution, Édimbourg, Scottish Government.

Scottish National Party, 2007, It's Time, Glasgow, The Montgomery Litto Group.

—, 2011, Re-elect a Scottish Government Working for Scotland, Édimbourg, Scottish National Party.

Scottish Parliament, 2008, Who Represents Me?, Édimbourg, Scottish Parliament.

Scottish Parliament Information Centre, 10 mai 2011, SPICe Briefing 11/29: Election 2011, Édimbourg, Scottish Parliament. Disponible sur <www.scottish.parliament.uk/ResearchBriefingsAndFactsheets/ S4/SB_11-29.pdf>.

Sturgeon Nicola, 2012 (3 décembre), «Bringing the powers home to build a better Scotland», Glasgow, Strathclyde University. Disponible sur <www.gov.scot/News/Speeches/better-nation-031212>. 
—, 2013 (25 janvier), «Scotland and Europe», Dublin, British-Irish Chamber of Commerce. Disponible sur <www.snp.org/blog/post/ 2013/jan/sturgeons-dublin-speech-scotland-and-europe>.

\section{Sources secondaires}

Anderson Benedict, 1990, Imagined Communities: Reflections on the Origin and Spread of Nationalism, Londres, Verso.

Billig Michael, 1995, Banal Nationalism, Londres, Sage.

BRAdBuRY Jonathan, 2006, «Territory and Power Revisited: Theorising Territorial Politics in the United Kingdom after Devolution», Political Studies, vol. 54, p. 559-582.

Brown Alice, MaCrone David \& Paterson Lindsay, 1998 [1996], Politics and Society in Scotland, Londres, Palgrave Macmillan, $2^{\mathrm{c}}$ éd.

Camp-Pietrain Edwige, 2008, «Les pétitions au Parlement écossais : une nouvelle pratique dans les coulisses du pouvoir», Observatoire de la société britannique, $\mathrm{n}^{\circ}$ 6, p. 177-195. Disponible sur <http:// osb.revues. org/464>.

—, 2014, L'Écosse et la tentation de l'indépendance: le référendum d'autodétermination de 2014, Villeneuve d'Ascq, Presses universitaires du Septentrion.

CraIg Cairns, 1999, The Modern Scottish Novel: Narrative And The National Imagination, Édimbourg, Edinburgh University Press.

Civardi Christian, 1998, L'Écosse depuis 1528, Paris-Gap, Ophrys.

CRAWFord Robert, 2014, Bannockburns: Scottish Independence and the Literary Imagination, 1314-2014, Édimbourg, Edinburgh University Press.

Curtice John \& Steven Martin, 2011, The 2011 Scottish Parliament Election: In-depth, Édimbourg, Electoral Reform Society.

Curtice John, MaCrone David, MaEwen Nicola, Marsh Michael \& Ormston Rachel, 2009, Revolution or Evolution? The 2007 Scottish Elections, Édimbourg, Edinburgh University Press.

Duclos Nathalie, 2009, «La démocratie britannique face au modèle parlementaire écossais», E-rea, vol. 7, n 1 . Disponible sur <http://erea. revues.org/861>.

—, 2013 (14 juin), «The Scottish Parliament Election of May 2011: Internal Factors in the SNP's Victory », Collection Individu et Nation, vol. 5. Disponible sur <http://revuesshs.u-bourgogne.fr/individu\&nation/ document.php?id $=657>$.

—, 2014, L'Écosse en quête d'indépendance? Le référendum de 2014, Paris, Presses de l'université Paris-Sorbonne.

FinLAY Richard, 1997, «Heroes, Myths and Anniversaries in Modern Scotland», Scottish Affairs, no 18, p. 108-125.

Keating Michael, 2001 [1996], Nations against the State: The New Politics 
of Nationalism in Quebec, Catalonia and Scotland, Basingstoke, Palgrave MacMillan, $2^{\mathrm{e}}$ éd.

LEYDIER Gilles, 2013 (17 juin), "It was not supposed to happen” : une mise en perspective historique des élections au Parlement écossais de mai $2011 »$, Collection Individu et Nation, vol. 5. Disponible sur <http:// revuesshs.u-bourgogne.fr/individu\&nation/document.php?id= 709>.

Lynch Peter, 2002, SNP: The History of the Scottish National Party, Cardiff, Welsh Academic Press.

Moffat Alexander \& Riach Alan, 2014, Arts of Independence, Édimbourg, Luath Press Ltd.

PAAsi Anssi, 1995, «Constructing Territories, Boundaries and Regional Identities», dans T. Forsberg (éd), Contested Territory. Border Disputes at the Edge of the Former Soviet Empire, Aldershot, Edward Elgar, p. 42-61.

Penrose Jan, 2002, "Nations, States and Homelands: Territory and Territoriality in Nationalist Thought», Nations and Nationalism, vol. 8, $\mathrm{n}^{\circ} 3$, p. 277-297.

Reicher Stephen, Hopkins Nick \& Harrison Kate, 2009, «Identity Matters: On the Importance of Scottish Identity for Scottish Society», dans F. Bechhofer et D. McCrone (éds), National Identity, Nationalism and Constitutional Change, Basingstoke, Palgrave Macmillan, p. 17-40.

SAck Robert, 1986, Human Territoriality: Its Theory and History, Cambridge, Cambridge University Press.

Thomsen Robert, 2010, Nationalism in Stateless Nations: Images of Self and Other in Scotland and Newfoundland, Édimbourg, John Donald. 